\title{
Cutaneous Cholesterol Embolization to the Lower Trunk: An Underrecognized Presentation
}

\author{
Nooshin K. Brinster, MD; Mark Levy, MD; Farah Awadalla, MD
}

\section{PRACTICE POINTS}

- Cholesterol embolization may occur in proximal locations, and index of suspicion should be high in patients who are at risk.

- Several biopsies may be necessary to make a diagnosis of cholesterol emboli.

To the Editor:

A 65-year-old man with severe atherosclerotic disease developed multiple painful eschars on the lower abdomen, thighs, sacrum, and perineum. He initially presented with myocardial ischemia and claudication and underwent 3 cardiac catheterizations as well as stenting of the superficial femoral artery. Within 2 weeks, he developed exquisitely tender nodules on the lower abdomen, clinically presumed to be sites of enoxaparin injections. These lesions gradually expanded and ulcerated to involve the sacrum, buttock, perineum, and upper thighs (Figure 1). Two punch biopsies from ulcerated skin taken 10 days apart demonstrated necrosis of skin and subcutaneous fat without evidence of vasculitis, vasculopathy, emboli, or notable inflammation despite examination of multiple levels of all submitted tissue. A definitive cause for the ulcerations remained elusive with development of new lesions. A third incisional biopsy of a newly developed, nonulcerated, subcutaneous nodule performed 8 weeks after presentation revealed multiple cholesterol emboli (Figure 2). He was treated with warfarin and clopidogrel bisulfate as well as local wound care. The lesions slowly resolved over the next 4 to 6 months.

Cholesterol embolization syndrome occurs when disrupted atherosclerotic plaques embolize from large proximal arteries to more distal arterioles, resulting in ischemic damage to 1 or more organ systems. ${ }^{1}$ It can occur spontaneously but often is a consequence of thrombolytic therapy, anticoagulation, and angioinvasive procedures. ${ }^{2,3}$ Cutaneous manifestations include livedo reticularis, retiform purpura, nodules, and gangrene. Although livedo reticularis may extend from the legs to the trunk, gangrenous lesions predominantly involve the distal digits.

This case illustrates the challenge in diagnosis of cholesterol emboli, both clinically and histologically. Cutaneous lesions are morphologically variable and often occur with systemic manifestations, mimicking numerous conditions. ${ }^{1}$ Lower extremity involvement is a wellknown occurrence in cholesterol embolization (ie, blue toe syndrome); however, periumbilical and lumbosacral

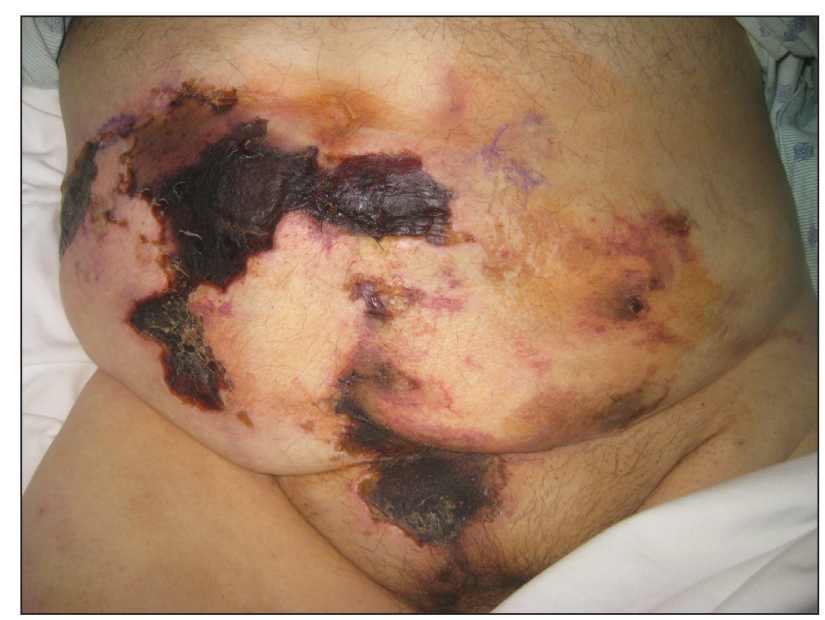

FIGURE 1. Cutaneous eschars. Geographic eschars on the lower abdomen and mons region.

From Virginia Commonwealth University, Richmond. Dr. Brinster is from the Departments of Dermatology and Pathology; Dr. Levy is from the Department of Surgery, Division of Vascular Surgery; and Dr. Awadalla is from the Department of Dermatology. 


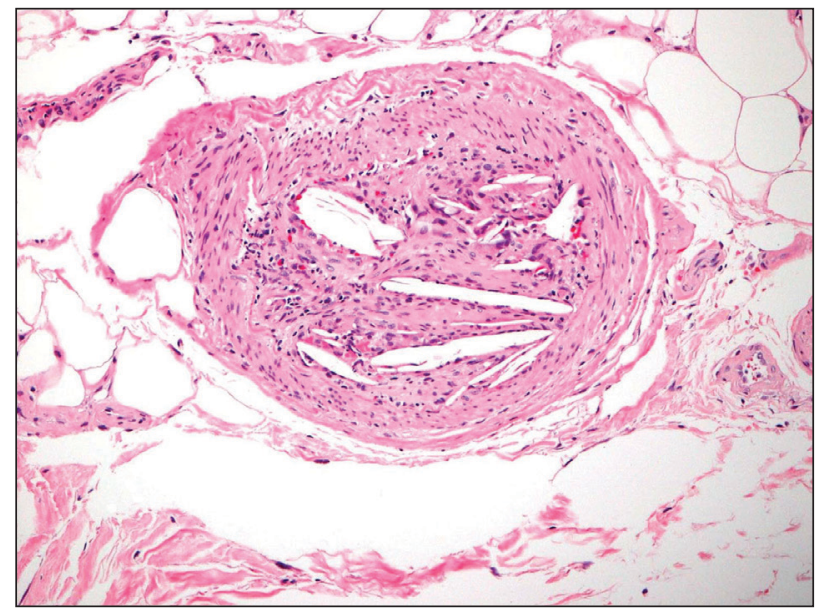

FIGURE 2. Cholesterol embolization. Histopathology revealed cholesterol clefts in an arteriole at the junction of the reticular dermis and subcutaneous fat $(H \& E$, original magnification $\times 400)$.

lesions have not been emphasized in the dermatologic or peripheral vascular literature. Our patient's initial diagnosis was enoxaparin necrosis at abdominal injection sites; however, this unusual distribution of lesions was ultimately determined to be the consequence of cholesterol embolization from the inferior epigastric and superficial external pudendal arteries at the time of stenting of the superficial femoral artery. Proximal truncal involvement should be recognized as an atypical but important cutaneous manifestation to facilitate timely diagnosis and treatment. ${ }^{4,5}$

Our patient's course also highlights the potential need for multiple biopsies. Although the gold standard for diagnosis is histologic confirmation, a negative biopsy does not always exclude cholesterol emboli, and one should have a low threshold to perform additional biopsies in the appropriate clinical setting.

\section{REFERENCES}

1. Fine MJ, Kapoor W, Falanga V. Cholesterol crystal embolization: a review of 221 cases in the English literature. Angiology. 1987;38:769-784.

2. Fukumoto $\mathrm{Y}$, Tsutsui $\mathrm{H}$, Tsuchihashi $\mathrm{M}$, et al. The incidence and risk factors of cholesterol embolization syndrome, a complication of cardiac catheterization: a prospective study. J Am Coll Cardiol. 2003;42:211-216.

3. Karalis DG, Chandrasekaran K, Victor MF, et al. Recognition and embolic potential of intraaortic atherosclerotic debris. J Am Coll Cardiol. 1991;17:73.

4. Zaytsev P, Miller K, Pellettiere EV. Cutaneous cholesterol emboli with infarction clinically mimicking heparin necrosis-a case report. Angiology. 1986;37:471-476.

5. Erdim M, Tezel E, Biskin N. A case of skin necrosis as a result of cholesterol crystal embolisation. J Plast Reconstr Aesthet Surg. 2006;59:429-432. 